\title{
Brain Exercising Games With Consumer-Grade Single-Channel Electroencephalogram Neurofeedback: Pre-Post Intervention Study
}

Pasin Israsena $^{1 *}, \mathrm{PhD}$; Suwicha Jirayucharoensak ${ }^{1 *}, \mathrm{PhD}$; Solaphat Hemrungrojn ${ }^{2,3 *}, \mathrm{MD}$; Setha Pan-Ngum ${ }^{4 *}, \mathrm{PhD}$

\footnotetext{
${ }^{1}$ National Electronics and Computer Technology Center, National Science and Technology Development Agency, Pathumthani, Thailand

${ }^{2}$ Department of Psychiatry, Faculty of Medicine, Chulalongkorn University, Bangkok, Thailand

${ }^{3}$ Cognitive Fitness Research Center, Chulalongkorn University, Bangkok, Thailand

${ }^{4}$ Department of Computer Engineering, Faculty of Engineering, Chulalongkorn University, Bangkok, Thailand

*all authors contributed equally
}

Corresponding Author:

Pasin Israsena, $\mathrm{PhD}$

National Electronics and Computer Technology Center

National Science and Technology Development Agency

112 Thailand Science Park

Klong Luang

Pathumthani, 12120

Thailand

Phone: 6625646900 ext 2471

Email: pasin.israsena@nectec.or.th

\section{Abstract}

Background: The aging population is one of the major challenges affecting societies worldwide. As the proportion of older people grows dramatically, so does the number of age-related illnesses such as dementia-related illnesses. Preventive care should be emphasized as an effective tool to combat and manage this situation.

Objective: The aim of this pilot project was to study the benefits of using neurofeedback-based brain training games for enhancing cognitive performance in the elderly population. In particular, aiming for practicality, the training games were designed to operate with a low-cost consumer-grade single-channel electroencephalogram (EEG) headset that should make the service scalable and more accessible for wider adoption such as for home use.

Methods: Our training system, which consisted of five brain exercise games using neurofeedback, was serviced at 5 hospitals in Thailand. Participants were screened for cognitive levels using the Thai Mental State Examination and Montreal Cognitive Assessment. Those who passed the criteria were further assessed with the Cambridge Neuropsychological Test Automated Battery (CANTAB) computerized cognitive assessment battery. The physiological state of the brain was also assessed using 16-channel EEG. After 20 sessions of training, cognitive performance and EEG were assessed again to compare pretraining and posttraining results.

Results: Thirty-five participants completed the training. CANTAB results showed positive and significant effects in the visual memory (delayed matching to sample [percent correct] $P=.04$ ), attention (median latency $P=.009$ ), and visual recognition (spatial working memory [between errors] $P=.03$ ) domains. EEG also showed improvement in upper alpha activity in a resting state (open-eyed) measured from the occipital area $(P=.04)$, which similarly indicated improvement in the cognitive domain (attention).

Conclusions: Outcomes of this study show the potential use of practical neurofeedback-based training games for brain exercise to enhance cognitive performance in the elderly population.

(JMIR Serious Games 2021;9(2):e26872) doi: 10.2196/26872

\section{KEYWORDS}

neurofeedback; serious gaming; serious game; brain exercise; cognition training; EEG; aging; cognition; cognitive; brain game 


\section{Introduction}

\section{Background}

The global population aged above 60 years is expected to increase from 900 million in 2015 to 1.4 billion by 2030 and to 2.1 billion by 2050 [1]. Approximately two-thirds of these elderly people live in low-income and middle-income countries [2]. This growing number of elderly will lead to an increase in the incidence of aging-related disorders, including cognitive-related diseases such as dementia, Alzheimer disease, and mild cognitive impairment (MCI), which are increasingly becoming more common [3]. Dementia, the most frequent brain disorder, is differentiated according to the etiology of either vascular dementia or neurodegenerative dementia (such as Alzheimer disease) [4,5]. Dementia is characterized by a devastating reduction in cognitive abilities, including control of behavior, learning, memory, attention/sleep, language, intelligence, perception, as well as functional independence and social relationships. More than 47 million people are living with dementia [6], approximately $60 \%$ of whom live in low- and middle-income countries. Driven by population aging, this number is expected to triple by 2050, as the incidence of dementia rises sharply at ages above 75 years. The economic impact has been estimated at US $\$ 600$ billion per year worldwide [7], including a significant burden of unpaid family caregiving.

In contrast to Alzheimer disease and dementia, which are conditions that are diagnosed at a state that is difficult to treat, MCI-representing an intermediate state between cognitive decline associated with normal aging and dementia [8,9]-is more suitable for targeted preventive measures along with active prevention in the healthy elderly population, as an elderly individual with MCI still has well-preserved functional abilities $[10,11]$. The global prevalence of MCI in the population aged above 60 years reaches up to $38.60 \%$ [12]. MCI is a risk factor for dementia [13] and is associated with a 6-fold increased risk of Alzheimer disease [14]. Indeed, as pharmaceutical-based treatments have yet to be very successful in a late-stage condition such as Alzheimer disease, and may come with certain side effects [15-18], researchers have been exploring the usefulness of alternative strategies to prevent, prolong decline, or even enhance cognitive performance with different methods such as cognitive training [19-22] or neurofeedback training (NFT) [23-25]. Because of the aging trend in developing countries mentioned above, in addition to their demonstrated effectiveness, these methods should be low cost so that they can be beneficial to the majority of the target group.

\section{NFT as a Solution for Cognitive Decline}

NFT is a noninvasive technique to alter brain activity [23-25]. NFT does not actively interfere with the brain but is rather a process during which subjects learn to influence their brain wave pattern by receiving feedback of their brain activity (visual, audio, or other modalities). The controllability of brain rhythms from users often also elicits neural plasticity in the brain that can affect their behaviors and cognitive functions. The use of NFT in medical and therapeutic contexts has continuously attracted interest in research and practice [23]. In particular, in the field that addresses mental problems such as attention deficit hyperactivity disorder (ADHD) or depression [26-31], NFT has been considered a potentially viable tool.

There are several types of NFT based on different brain imaging technologies such hemoencephalographic, low-resolution electromagnetic tomography, functional magnetic resonance imaging, or functional near-infrared spectroscopy. However, the most common type of NFT usually provides brain state information to the user via electroencephalogram (EEG) signals (also called EEG biofeedback). Recorded at the scalp, the EEG is produced by synchronous postsynaptic potentials from thousands to millions of neurons. When amplified, digitized, and plotted, the raw EEG signal appears as a complex oscillatory pattern. Raw EEG signals are observed via frequency bands of interest (ie, delta, 1-4 Hz; theta, 4-8 Hz; alpha, 8-12 Hz; beta, $12-32 \mathrm{~Hz}$; and gamma, 32-60 Hz), usually in the forms of their amplitudes or power spectra.

Brain wave characteristics have been shown to change dramatically depending on the task at hand. Among these, alpha is a particularly interesting oscillation, which is the predominant rhythm in the human brain in a resting state, especially when the eyes are closed [32]. Originally, alpha NFT was considered as simple relaxation training. However, renewed interest has arisen to clarify the relation between the alpha frequency band and cognitive performance [33-35] via indicators such as alpha peak frequency [36,37] and individual upper alpha amplitude/power [38-40]. In resting-state recordings, the alpha peak is clearly visible between 7.5 and $12.5 \mathrm{~Hz}$. Higher alpha peak frequencies (eg, $12 \mathrm{~Hz}$ in comparison to $10 \mathrm{~Hz}$ ) have been shown to correlate positively with high memory performance $[36,37]$ and IQ [41]. Moreover, in terms of alpha amplitude or power, a positive correlation between individual upper alpha amplitude and IQ has been reported (eg, [42,43]). Additionally, as suggested by Klimesch [38], high alpha power during a resting state and low alpha power during the execution of a task were associated with good performance in semantic long-term memory tasks. Despite these promising results, NFT has not yet been translated into mainstream practice. This could be due to limitations of study designs such as lack of a methodological design, therapeutic effect, and validity, as reviewed previously $[44,45]$.

\section{Consumer-Grade EEG}

Until recently, most EEG equipment has been designed for either research or medical purposes. These devices were rather expensive and not easy to operate. An EEG headset usually has multichannel EEG wet sensors held together with a head cap in predefined positions according to the international 10-20 system [46]. Therefore, considerable time is needed for each setup before use and for subsequent cleanup, as these wet sensors needed to be lubricated before each use. The emergence of low-cost consumer-grade EEG headsets offers an easy-to-administer and practical solution via the use of dry or semidry electrodes, along with wireless connectivity, thereby opening the technology for mass use. More importantly, most of the consumer-grade EEGs also have an option for continuous raw EEG reading, as opposed to the batch records usually 
offered in medical-grade EEGs. This online EEG reading feature is a necessity for systems such as NFT.

There are several consumer-grade EEGs currently available on the market, which differ in terms of the numbers and types of electrodes, positioning, and design. Prominent examples include the Neurosky, Emotiv, or Muse systems. Researchers have been evaluating and comparing these devices with research- or medical-grade solutions [47-52]. In general, the research- or clinical-grade EEGs are more accurate as they employ multichannel wet sensors. For consumer-grade EEG, studies have confirmed that useful EEG readings are possible. These devices are easy to use, but involve dry electrodes with no impedance matching, making them more susceptible to artifacts. They also generally come with a lower number of channels.

Consumer-grade EEG-based systems for applications such as NFT for children with ADHD have already been discussed (eg, [29,30]). We have also published primary research results of NFT using Emotiv [53]. Experience from the trial indicated that although the multichannel Emotiv system provided good results, it was quite time-consuming to administer, as saline still needed to be applied for each sensor. The headset design was also found to be rather bulky for long-term serious use, especially if it was to be shared as part of a multiuser training station.

Alternatively, a single-channel device would provide an even cheaper solution that could expand deployment sites. In particular, the Neurosky design appeared to offer a solid headset design with a single dry electrode placed on the forehead. Although Maskeliunas et al [51] compared the Neruosky device with the multichannel Emotiv device and found the reading quality to be somewhat inferior, it was hypothesized that for NFT application, Neurosky could still be sufficient, especially as the position of the sensor is outside the frontal lobe area, where the cognitive state of interest such as attention occurs.
Indeed, in contrast to the results of Maskeliunas et al [51], some authors have gone as far as stating that a single-channel device was preferred [45]. This was supported by the finding of a negative correlation between the number of bands used to compose the feedback signal and the success of training; that is, more complicated protocols that promoted or inhibited several bands worsened the final results.

The aim of this study was therefore to implement a system of brain training games with NFT on a single channel using a consumer-grade EEG headset, and to evaluate its efficacy for cognitive training in elderly participants. Physiological evidence in terms of EEG was also investigated to determine whether the brain rhythms of interest change as expected after training.

\section{Methods}

\section{System Overview}

Overall, the architecture of the NFT gaming system was designed to support multiuser application in multiple locations. At each location, the PC-based games ran locally on the computer, with the Neurosky EEG headset connected via Bluetooth to provide brain signal feedback. Training data were collected and sent to the remote database server over the internet (Figure 1).

In terms of software, the games were developed using the Unity game development platform [54], and were managed by the Neurofeedback Cognitive Training Manager program written in C\#. Training data were delivered to the server via the RESTful application programming interface (API) web service. The server was programmed using PHP with the MySQL database. Parameters such as EEG readings, scoring, and usage time/characteristics of each user could be collected for analysis via the system's web service API (Figure 2).

Figure 1. System overview. API: application programming interface.

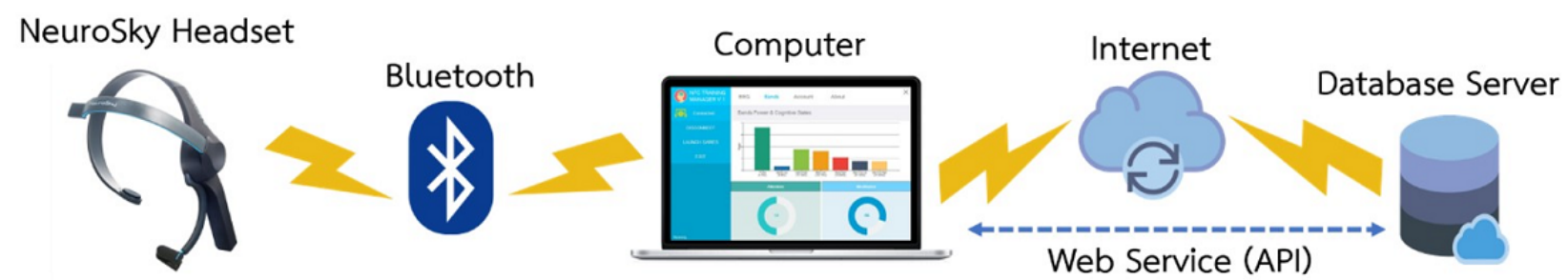


Figure 2. System configuration. NFCT: Neurofeedback Cognitive Training; API: application programming interface.

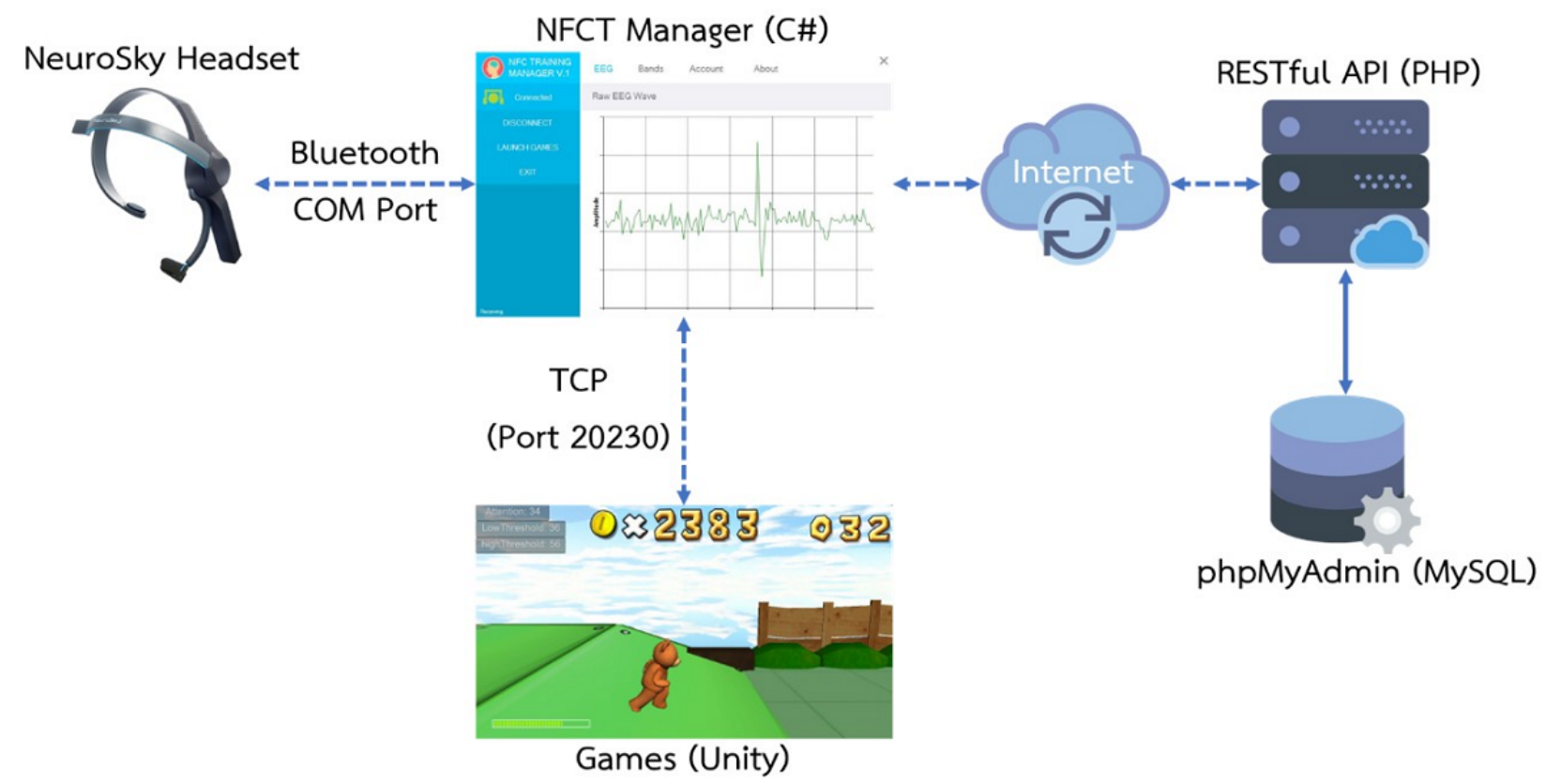

\section{NFT Control and EEG Signal Processing}

\section{Selection of Feedback Signals}

Different strategies for selecting which brain rhythms to be fed back for training had been considered, as discussed previously $[45,55]$. In general, there are two classical directions in NFT, focusing on either low frequencies (alpha or theta) to strengthen relaxation and focus or on high frequencies (low beta, beta, and theta) for reinforcing activation, organizing, and inhibiting distractibility. More specifically, the alpha protocol (ie, using the alpha wave as the feedback signal) has historically been used for relaxation training. Beta was considered to be associated with mental performance, and was used to improve focus or attention; alpha/theta has been used for stress reduction; gamma is a high-frequency band associated with cognitive processing and memory; and theta (including theta/beta) has been considered for anxiety, depression, ADHD, and emotional disorder treatments.

For this study, we decided on the type of feedback signals for our cognitive training scheme based on our observation that the strongest evidence in the cognitive training domain has been related to attention [26,29,30,53]. Attention was defined as the ability to focus cognitive resources on one relevant aspect of the environment while ignoring other irrelevant aspects. A significant portion of the published attention-based NFT literature suggests the use of alpha and beta rhythms [45,55-58]. For example, Li et al [58] provided evidence that the beta band (during a task) contained considerable information about attention, indicating the possibility of recognizing a subject's attention level by studying the EEG data. In addition, according to Klimesch [36], the alpha band showed task-related desynchronization; that is, it increased during resting states (especially when the eyes were closed) and decreased during performance of a cognitive task (eg, mental calculations). Therefore, it was reasonable to try to mimic the phenomena observed by the good performers by means of NFT (ie, to enhance beta power and reduce alpha power during active mental tasks) to enhance cognitive performance. Accordingly, our system targeted training of attention as the first cognitive function for improvement. Correlations between attention and other cognitive functions such as memory have previously been discussed. For example, memory showed limited capacity, and thus attention determined what would be encoded [59]. We hypothesized that improvement in higher attention could also lead to improvement in other cognitive domains.

In our previous work [53], the feedback was defined as the attention level calculated as:

$$
\text { Original attention feedback }=\mathrm{K}_{\beta} \times \mathrm{P}_{\beta} / \mathrm{K}_{\alpha} \times \mathrm{P}_{\alpha}
$$

Where $\mathrm{K}_{\beta}$ and $\mathrm{K}_{\alpha}$ are empirical scaling constants, $\mathrm{P}_{\beta}$ is the power spectral density (PSD) of the beta band (12-32 Hz), and $\mathrm{P}_{\alpha}$ is the PSD of the alpha band $(8-12 \mathrm{~Hz})$.

With recent studies showing that theta $(4-8 \mathrm{~Hz})$ training that could affect memory performance [38], with beta low (12-16 $\mathrm{Hz}$ ) specifically found to affect attention [60], we revised the attention feedback level as follows:

$$
\text { New attention feedback }=\left(\mathrm{K}_{\beta} \times \mathrm{P}_{\beta \mathrm{L}}\right) /\left(\mathrm{K}_{\alpha \mathrm{L}} \times \mathrm{P}_{\alpha \mathrm{L}}+\mathrm{K}_{\theta} \times \mathrm{P}_{\theta}\right)
$$

Where $\mathrm{P}_{\beta \mathrm{L}}$ is the PSD of the beta low band (12-16 Hz), $\mathrm{P}_{\alpha \mathrm{L}}$ is the PSD of the alpha low band $(8-10 \mathrm{~Hz})$, and $\mathrm{P}_{\theta}$ is the PSD of the theta band $(4-8 \mathrm{~Hz})$.

As the individual upper alpha (IUA) has been shown to affect cognitive power [38], the value of cognitive level monitored was then calculated from:

$$
\begin{aligned}
& \text { Cognitive level }=\quad\left(\mathrm{K}_{\beta} \times \mathrm{P}_{\beta \mathrm{L}}+\mathrm{K}_{\alpha \mathrm{h}} \times \mathrm{P}_{\alpha \mathrm{h}}\right) / \\
& \left(\mathrm{K}_{\alpha \mathrm{L}} \times \mathrm{P}_{\alpha \mathrm{L}}+\mathrm{K}_{\theta} \times \mathrm{P}_{\theta}\right)
\end{aligned}
$$

Where $\mathrm{P}_{\alpha \mathrm{h}}$ is the PSD of the alpha high band (10-12 Hz). 


\section{Adaptive Threshold Control}

In our system, the brain feedback signal (attention) was used as a game-controlling signal in an adaptive manner, with two additional parameters introduced: the upper and lower thresholds. Before each session, the system would measure the user's level of attention under two mental states: being attentive and relaxed. The difference between the two values was used to calculate the upper and lower threshold levels. Figure 3 shows an example of the condition when the lower and upper thresholds were adjusted by one third of the difference from the relaxed and attentive states, respectively. During the game play, if the user's attention level was higher than the threshold level, they were rewarded such as by having the controlled character moving faster. By contrast, if the attention level was lower than the lower threshold, the character would slow down. Attention levels between the two thresholds had no effects on the game being played. As the brain signal is highly volatile and is known to exhibit session-to-session variation, the value of the thresholds would continuously adapt to suit the present situation (Figure 4). For example, if the user became fatigued and the attention level dropped down for too long, the thresholds would adapt so that the user could hit the upper threshold more easily. This was important so that the user could feel that the game was responsive and therefore retain their engagement.

Figure 3. Setting upper and lower thresholds.

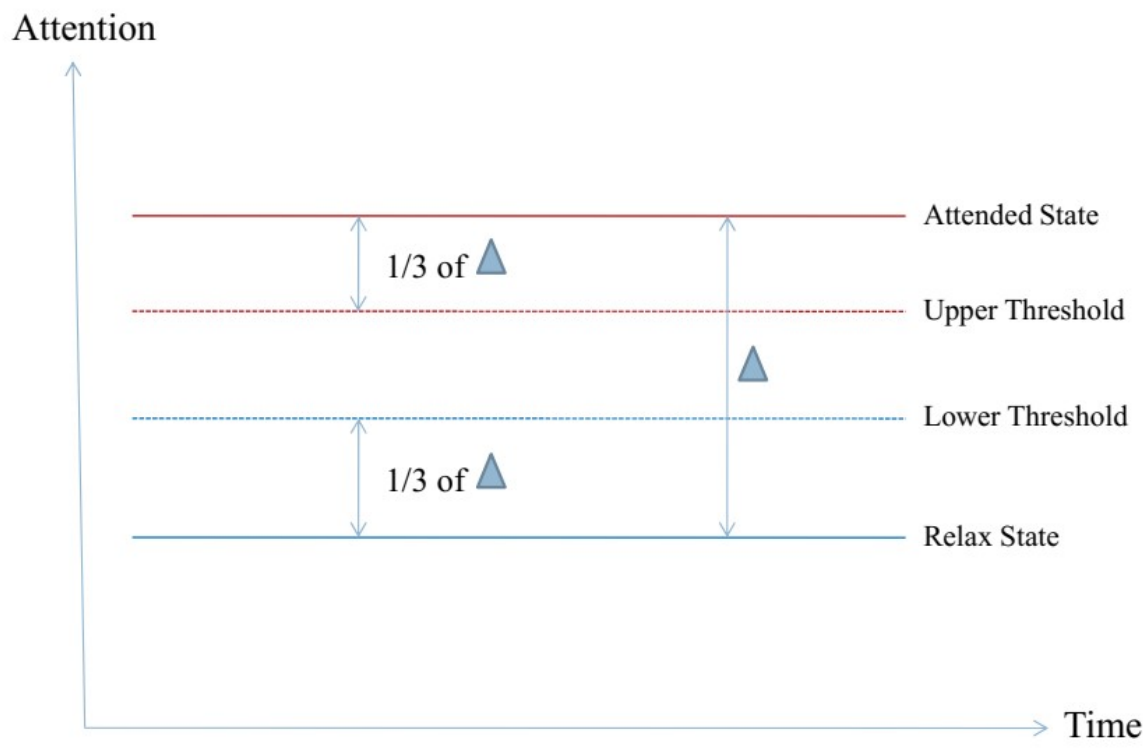

Figure 4. Adaptive threshold.

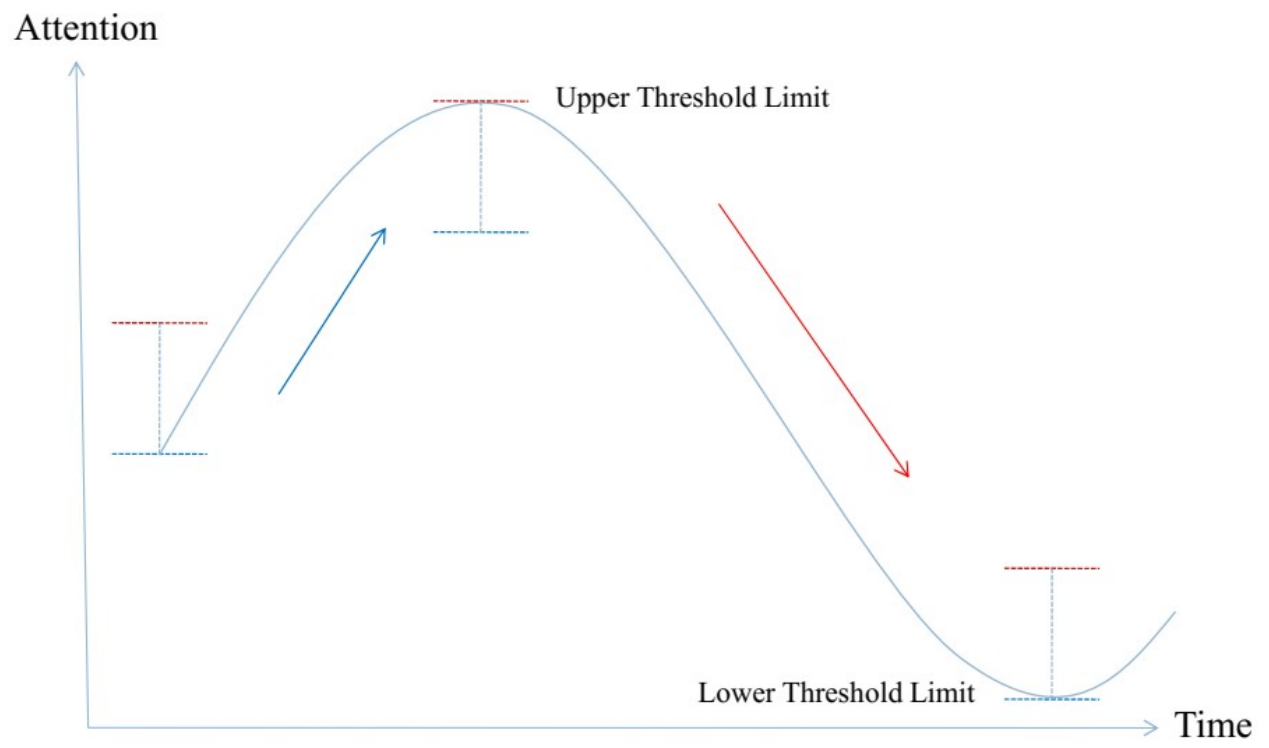

\section{Game Description}

In terms of game design, there were five games in our NFT system (Figure 5). The main concept used for all 5 games was based on the same idea that the main character's moving characteristic is controlled by the attention level feedback. The different scenarios between the games were mainly aimed at maintaining the user's engagement. In "Run Run," the first game, the bear runs faster when the player focuses on the game (ie, attention level above the upper threshold) and runs slower when the user loses focus (ie, attention below the lower 
threshold). The bear could also jump to collect coins if he ran fast enough, which was designed to provide the user more incentive to focus. For the second game, termed "Sunshine Day," if the player focused on the girl character to cheer her up, the girl in the garden would look happy and the character would jump to indicate that she's in a good mood. In "Cast Away," the third game, the player's attention level controls the cooking fire. There are three types of food, each of which requires a different level of cooking heat to be cooked. If the fire is strong, the food will be cooked quickly, resulting in high game scores. The fourth game was called "Paper Plane." If the attention value is high, the plane moves fast and is able to fly away from obstacles. Competing against paper planes that are computer-controlled was also provided for motivation. The last game was “321 Shoot." In this game, the average attention level was calculated every 3 seconds. If it was above the set threshold, then the basketball player scored. After every 10 points, the threshold was increased, and the basketball player would stand further away from the hoop.

Figure 5. Screenshots of games: (A) Run Run, (B) Sunshine Day, (C) Cast Away, (D) Paper Plane, (E) 321 Shoot.
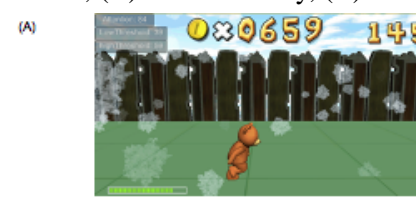

(B)

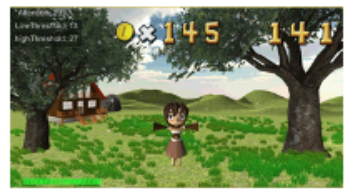

(C)

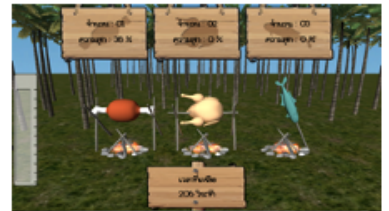

(D)
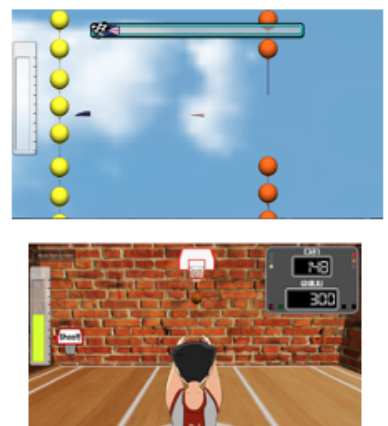
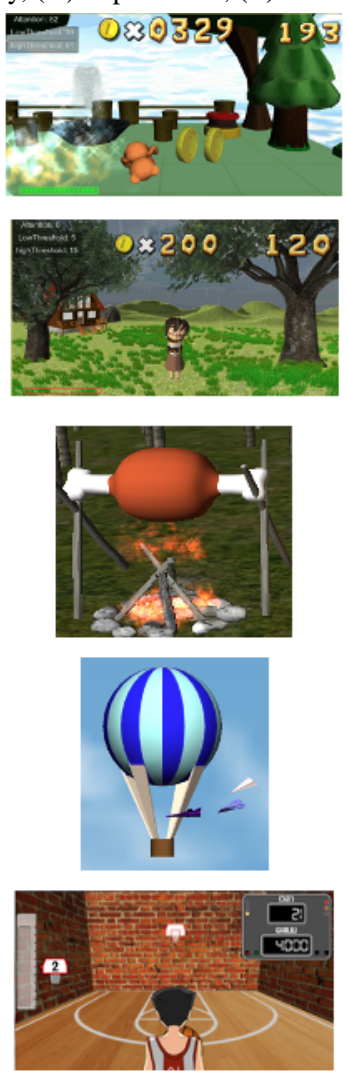

\section{Trial Protocol}

\section{Design and Setting}

The trial was designed as a multisite pilot study. Forty participants were targeted for the intervention by undergoing training at the following 5 participating hospitals and health centers in Thailand: King Narai Hospital; Queen Savang Vadhana Memorial Hospital; Thai Redcross Health Station 2, Sukuman Health Center; Thai Redcross Health Station 5, Sawangkanives; and Ban Bang Khae Social Welfare Development Center for Older Persons.

Intervention results were compared with those obtained from the control group, who received care-as-usual treatment.

\section{Participants and Eligibility}

Subjects were recruited on a voluntary basis from the participating sites. All subjects who had received a diagnosis of normal aging elderly and MCI were invited to participate. Diagnoses were made by psychiatrists according to standardized clinical criteria (Thai Mental State Examination [TMSE] and
Montreal Cognitive Assessment [MoCA]-Thai) and the criteria for MCI established by Petersen et al [11].

The inclusion criteria were 55-80 years old, with adequate verbal expression, visual and hearing abilities; at least 4 years of education; $\geq 24$ on the TMSE and $\leq 24$ on the MoCA-Thai for MCI patients, or $\geq 24$ on the TMSE and $>24$ on the MoCA-Thai for normal aging elderly; and not currently enrolled in another research study.

The exclusion criteria were presence or history of a confounding central neurologic disease (eg, brain tumor, stroke, epilepsy); currently under acetyl cholinesterase inhibitor treatment (eg, donepezil, galantamine, and rivastigmine); and presence of substance abuse disorder or substance dependence.

\section{Experimental Protocol}

At the pretest period, all test subjects were assessed for cognitive functions via Cambridge Neuropsychological Test Automated Battery (CANTAB) tests. The data, together with EEG records, were collected as baseline data. The intervention period started no later than 1 week after the assessment. The intervention group 
trained via NFT for attention for 30 minutes per session with 2 sessions per week for a period of 10 weeks (total 20 sessions). The subjects were then evaluated for cognitive functions outcome at week 10. Posttraining EEG data were also collected for analysis.

The study was approved by the Institutional Review Board of the Faculty of Medicine, Chulalongkorn University, Bangkok, Thailand (593/57). All participants provided written informed consent, and the study was performed in accordance with the Declaration of Helsinki.

\section{Cognitive Performance (CANTAB)}

\section{Delayed Matching to Sample}

Delayed matching to sample (DMS) was used to assess both simultaneous visual matching ability and short-term visual recognition memory for nonverbalizable patterns. Each user was shown a complex visual pattern (abstract and nonverbal), followed by four similar patterns after a brief delay $(0,4$, or 12 seconds). The pattern that exactly matched the sample had to be selected (Table 1).

Table 1. Parameters used for delayed matching to sample assessment.

\begin{tabular}{ll}
\hline Parameters & Meaning \\
\hline$\%$ correct & $\begin{array}{l}\text { Percentage of occasions upon which the subject selected the correct stimulus } \\
\text { Percentage of occasions upon which the subject selected the correct stimulus in trials when the } \\
\text { target stimulus and the three distractors were presented after the stimulus had been hidden, with } \\
\text { delays of } 0 \mathrm{~ms}\end{array}$ \\
$\begin{array}{l}\text { The percentage of occasions upon which the subject selected the correct stimulus in trials when } \\
\text { the target stimulus and the three distractors were presented after the stimulus had been hidden, } \\
\text { with delays of } 0 \text { ms, } 4000 \text { ms, and } 12,000 \text { ms }\end{array}$ \\
$\begin{array}{l}\text { Median latency considering all occasions where the subject selected the correct stimulus on their } \\
\text { first response in trials when the target stimulus and the three distractors were presented after the } \\
\text { stimulus had been hidden after a specified delay. Latency was measured in milliseconds; a lower } \\
\text { score was better }\end{array}$
\end{tabular}

Probability of an error occurring when the previous trial was responded to incorrectly

\section{Motor Screening Task}

The motor screening task (MOT) was used to assess whether sensorimotor deficits or lack of comprehension would limit the collection of valid data from the participant. For this test, colored crosses were presented in different locations on the screen, one at a time. The participant had to select the cross on the screen as quickly and accurately as possible (Table 2).

Table 2. Parameters in the motor screening task assessment.

\begin{tabular}{ll}
\hline Parameters & Meaning \\
\hline Mean latency & $\begin{array}{l}\text { Arithmetic mean of latency, defined as the time taken for the subject to touch the cross after it } \\
\text { appeared. Latency was measured in milliseconds; a lower mean was better } \\
\text { Median latency }\end{array}$ \\
Mean error & $\begin{array}{l}\text { Arithmetic median of latency } \\
\text { Measure of the subject's pointing accuracy, according to the mean distance between the center } \\
\text { of the cross and the location the subject touched on the screen, for the } 10 \text { crosses presented to } \\
\text { which the subject correctly responded; a lower error was better }\end{array}$
\end{tabular}

\section{Pattern Recognition Memory}

Pattern recognition memory was applied as a test of visual pattern recognition memory. The participant was presented with a series of visual patterns, one at a time, in the center of the screen. These patterns were designed so that they could not easily be given verbal labels. In the recognition phase, the participant was required to choose between a pattern they had already seen and a novel pattern in a 2-choice forced discrimination paradigm. In addition, the test patterns in this phase were presented in reverse order to the original order of presentation. This was then repeated, immediately or after a delay, with new patterns (Table 3).

Table 3. Parameters for the pattern recognition memory assessment.

\begin{tabular}{ll}
\hline Parameters & Meaning \\
\hline$\%$ correct & Number of correct responses, expressed as a percentage \\
Mean correct latency & Arithmetic mean of the correct responses; a lower value was better \\
Median correct latency & Arithmetic median of the correct responses; a lower value was better \\
$\#$ correct & Number of correct answers given by the subject \\
\hline
\end{tabular}




\section{Rapid Visual Information Processing}

The rapid visual information processing (RVP) assessment was designed to measure sustained attention. At a rate of 100 digits per minute, digits from 2 to 9 appeared in a pseudorandom order inside a white box shown at the center of the screen. Participants were requested to detect target sequences of digits (eg, 3-5-6). The button in the center of the screen had to be selected as quickly as possible when the target sequence was noted. The level of difficulty varied with either one- or three-target sequences that the participant had to watch for at the same time (Table 4).

Table 4. Parameters for rapid visual information processing assessment.

\begin{tabular}{ll}
\hline Parameters & Meaning \\
\hline $\mathrm{A}^{\prime}$ & How effective the subject was at detecting target sequences \\
Probability of hit & $\begin{array}{l}\text { The probability of the subject responding correctly; } \\
\text { a higher value was better }\end{array}$ \\
Total false alarms & $\begin{array}{l}\text { Number of times the subject responded outside the response window of a target sequence; a } \\
\text { lower value was better }\end{array}$ \\
Mean latency & Mean time taken to respond (milliseconds) \\
Median latency & $\begin{array}{l}\text { Measure of the median time taken to respond } \\
\text { Probability of false alarm }\end{array}$ \\
& $\begin{array}{l}\text { Probability of the subject responding inappropriately, equal to total false alarms/(total false alarms } \\
+ \text { total correct rejections); a lower value was better }\end{array}$ \\
\hline
\end{tabular}

\section{Spatial Span}

A spatial span test was used to assess visuospatial working memory capacity. White squares were shown on the screen, some of which briefly changed color in a variable sequence. The boxes that changed color in the same order in which they were displayed by the computer (for the forward variant) or in the reverse order (for the backward variant) had to be selected. The number of boxes in the sequence increased from two at the start of the test to nine at the end of the sequence, and the colors were varied throughout the test (Table 5).

Table 5. Parameters in the spatial span assessment.

\begin{tabular}{ll}
\hline Parameters & Meaning \\
\hline Span length & The longest sequence successfully recalled by the subject \\
Mean time to first response (span length 3) & $\begin{array}{l}\text { The time the subject took to initiate problems of the span length specified by the span length } \\
\text { option (3). The time was measured from the end of the presentation phase (the moment the final } \\
\text { box closed) until the subject touched the screen. Attempts undertaken on spans that the subject } \\
\text { did not pass were included in this calculation. A lower value was better. } \\
\text { Total errors }\end{array}$ \\
Mean time to last response (span length 3) & $\begin{array}{l}\text { Time the subject took to complete problems of the span length (3). The time was measured from } \\
\text { the end of the presentation phase (the moment the final box closes) to the time of the subject's } \\
\text { final response on a given attempt. Attempts undertaken on spans that the subject did not pass } \\
\text { were included in this calculation. A lower value was better. }\end{array}$ \\
\hline
\end{tabular}

\section{Spatial Working Memory}

The spatial working memory (SWM) test required the retention and manipulation of visuospatial information, and provided a measure of strategy as well as working memory errors. First, several colored squares (boxes) were shown on the screen. By selecting the boxes and using a process of elimination, the participant had to find one yellow "token" in each of a given number of boxes and use them to fill up an empty column on the righthand side of the screen. The number of boxes could be gradually increased until a maximum of 12 boxes were shown for the participants to search. The color and position of the boxes used also changed from trial to trial to discourage the use of stereotyped search strategies (Table 6). 
Table 6. Parameters for spatial working memory (SWM) assessment.

\begin{tabular}{ll}
\hline Parameters & Meaning \\
\hline Between errors & $\begin{array}{l}\text { Results for trials containing the number of boxes specified by } \mathrm{n}(4,6, \text { or 8) only; a lower value } \\
\text { was better }\end{array}$ \\
Strategy & $\begin{array}{l}\text { Specifies the lower and upper numbers of boxes to calculate the SWM strategy measure; a lower } \\
\text { value was better } \\
\text { Median time to first response }\end{array}$ \\
$\begin{array}{l}\text { Mean time between the problem being presented to the subject and the subject first touching the } \\
\text { screen to open a box, for problems with the specified number of boxes; a lower value was better } \\
\text { Median time to last response }\end{array}$ & $\begin{array}{l}\text { Mean time of the subject's last response for a problem, calculated from the time between the } \\
\text { problem being presented to the subject and the subject's last screen touch to open a box to locate } \\
\text { the final token for the problem; a lower value was better }\end{array}$ \\
\hline
\end{tabular}

\section{Physiological Brain Characteristics}

Before the first training session, each participant recoded their brain EEG signals using a research-grade multichannel g.Nautilus headset from g.tec medical engineering. The g.Nautilus device is a wireless EEG sensing and amplification system with a 24-bit resolution at a $250 / 500 \mathrm{~Hz}$ sampling rate. The version used was configured to work with g.SAHARA hybrid EEG electrodes for dry or wet recordings. After asking the participant to relax, the EEG signals were recorded at 16 positions (Fp1, Fp2, F3, Fz, F4, T7, C3, Cz, C4, T8, P2, Pz, P4, $\mathrm{PO}$, PO8, and Pz) when the eyes were both open and closed. The entire procedure was repeated after the last training session (session 20).

\section{Results}

\section{Participants}

A total of 104 individuals applied for the intervention program. Seventeen were excluded from the study based on their health conditions. A further 22 candidates did not meet the TMSE and MoCA criteria. Of the remaining candidates, 43 individuals eventually participated in the program and 35 completed the training (8 dropouts).

\section{CANTAB Results}

Table 7 shows the baseline CANTAB values of the control and intervention groups. Analysis results of CANTAB readings between pre- and posttraining using paired $t$ tests are shown in Table 8 .

Statistically significant improvements were found in terms of DMS (\%correct, \%correct all delays), median latency, and SWM (between errors), meaning that there were benefits from the training in terms of visual memory (DMS), attention (MOT), and visual recognition (SWM). Improvements were also found in the control group in terms of RVP (probability of hit, probability of false alarm) and MOT (median latency, mean error).

The results indicated that after brain exercising based on the proposed NFT system, we did find improvements in both memory and attention domains as we had hoped. DMS was the test of visual matching to sample, assessing mainly visual memory, and depended heavily on a forced-choice decision that may be dependent on frontal as well as temporal lobe functions. By contrast, the SWM score depended on the ability to retain spatial information to manipulate remembered items in working memory. Therefore, the improved SWM score could indicate improvements in the frontal lobe as well as in executive function.

Table 7. Baseline information of the participants.

\begin{tabular}{lll}
\hline Characteristic & Control $(\mathrm{n}=30)$ & Neurofeedback training $(\mathrm{n}=35)$ \\
\hline Age (years), mean (SD) & $69.86(8.95)$ & $69.48(6.15)$ \\
Female, n (\%) & $28(93)$ & $27.09(2.05)$ \\
TSME $^{\mathrm{a}}$, mean (SD) & $27.50(1.70)$ & $21.00(2.03)$ \\
MoCA $^{\mathrm{b}}$, mean (SD) & $20.83(2.53)$ & \\
\hline
\end{tabular}

aTMSE: Thai Mental State Examination.

${ }^{\mathrm{b}}$ MoCA: Montreal Cognitive Assessment. 
Table 8. Cambridge Neuropsychological Test Automated Battery results.

\begin{tabular}{|c|c|c|c|c|c|c|}
\hline \multirow[t]{2}{*}{ Parameter $^{\mathrm{a}}$} & \multicolumn{3}{|l|}{ Control } & \multicolumn{3}{|c|}{ Neurofeedback training } \\
\hline & $t(d f=29)$ & $\mathrm{SD}(\mathrm{x}-\mathrm{y})$ & $P$ value & $t(d f=34)$ & $\mathrm{SD}(\mathrm{x}-\mathrm{y})$ & $P$ value \\
\hline \multicolumn{7}{|l|}{$\mathbf{D M S}^{\mathrm{b}}$} \\
\hline$\%$ correct & -0.5177 & 8.8165 & .61 & -3.0566 & 9.2627 & .004 \\
\hline \%correct (simultaneous) & 1.2289 & 10.4 & .23 & -1.5082 & 14.5695 & .14 \\
\hline$\%$ correct (all delays) & -0.9581 & 11.4336 & .35 & -2.6199 & 11.6131 & .01 \\
\hline Median correct latency & -1.9927 & $1.02 \mathrm{E}+03$ & .06 & 0.3146 & $1.18 \mathrm{E}+03$ & .76 \\
\hline Prob error given error & -0.4167 & 0.2088 & .68 & 0.7981 & 0.1566 & .43 \\
\hline \multicolumn{7}{|l|}{ MOT $^{\mathbf{c}}$} \\
\hline Mean latency & 1.8352 & 244.0492 & .08 & 1.0251 & 275.8327 & .31 \\
\hline Median latency & 3.6956 & 166.6858 & $<.001$ & 2.7713 & 213.8717 & .009 \\
\hline Mean error & -2.5181 & 2.7958 & .02 & -0.9254 & 5.1444 & .36 \\
\hline \multicolumn{7}{|l|}{ PRM $^{d}$} \\
\hline$\%$ correct & -0.1606 & 9.472 & .87 & -1.1758 & 14.9745 & .25 \\
\hline Mean correct latency & -0.5861 & 596.2864 & .56 & 0.8479 & $1.18 \mathrm{E}+03$ & .40 \\
\hline Median correct latency & -1.6612 & 248.2209 & .11 & 1.4163 & 502.0288 & .17 \\
\hline \#correct & -0.1575 & 2.3183 & .88 & -1.1758 & 3.5939 & .25 \\
\hline \multicolumn{7}{|l|}{$\mathbf{R V P}^{\mathbf{e}}$} \\
\hline $\mathrm{A}^{\prime}$ & -2.041 & 0.0535 & .05 & -0.3157 & 0.0802 & .75 \\
\hline Probability of hit & -2.3338 & 0.1766 & .03 & 0.5545 & 0.1806 & .58 \\
\hline Total false alarms & 0.3692 & 1.978 & .71 & 0.8318 & 7.5184 & .41 \\
\hline Mean latency & -0.1099 & 90.2135 & .91 & 1.6845 & 265.2661 & .10 \\
\hline Median latency & 0.2864 & 110.6188 & .78 & 1.9901 & 289.9302 & .05 \\
\hline Probability of false alarm & 2.2401 & 0.0083 & .03 & 0.8618 & 0.0325 & .39 \\
\hline \multicolumn{7}{|l|}{$\operatorname{SSP}^{\mathbf{f}}$} \\
\hline Span length & 1.27 & 1.0063 & .21 & -1.0964 & 1.0792 & .28 \\
\hline Mean time to first response (span length 3) & -0.9127 & 622.248 & .37 & 1.2085 & $1.50 \mathrm{E}+03$ & .24 \\
\hline Total errors & 0.1436 & 6.3576 & .89 & 0.2642 & 7.0368 & .79 \\
\hline Mean time to last response (span length 3) & 1.5412 & $1.32 \mathrm{E}+03$ & .13 & 1.0786 & $2.43 \mathrm{E}+03$ & .29 \\
\hline \multicolumn{7}{|l|}{ SWM ${ }^{\mathbf{g}}$} \\
\hline Between errors & -0.2158 & 9.3051 & .83 & 2.2582 & 12.2757 & .03 \\
\hline Strategy & 0.5781 & 2.8424 & .57 & 0.0838 & 4.0362 & .93 \\
\hline Median time to first response & -1.5109 & 654.9549 & .14 & 0.7888 & 991.3451 & .44 \\
\hline Median time to last response & -0.6447 & $5.89 \mathrm{E}+03$ & .52 & 1.1679 & $7.14 \mathrm{E}+03$ & .25 \\
\hline
\end{tabular}

${ }^{\mathrm{a}}$ See Tables 1-6 for a description of the parameters.

${ }^{b}$ DMS: delayed matching to sample.

${ }^{\mathrm{c}}$ MOT: motor screening.

${ }^{\mathrm{d}}$ PRM: pattern recognition memory.

${ }^{\mathrm{e}} \mathrm{RVP}$ : rapid visual information processing.

${ }_{\text {fSP: spatial span. }}$

${ }^{\text {g }}$ SWM: spatial working memory. 


\section{EEG Results}

To analyze the EEG data, the channels were first grouped according to their cortical areas, as shown in Figure 6. This was necessary because of the known associations between brain patterns and where they would occur in the brain areas: the prefrontal (Group 1), occipital (Group 2), frontal (Group 3), central (Group 4), and parietal (Group 5) areas. Signals from the prefrontal and frontal areas (Group 6) and from the parietal and occipital areas (Group 7) were also analyzed together, respectively. EEGs from the temporal left and right sides were grouped as Group 8 and Group 9, respectively.
The PSD of each group was then calculated as the average of each channel's PSD within the group, with the paired $t$ test calculated from pretraining and posttraining group PSD values. Significant changes in alpha high were found during the open-eyed relaxed condition in Group 2 and Group 7 ( $P=.04$ for both). This agreed well with the finding of Klimesch [38] that an increase in alpha high corresponded to improvement in cognitive performance. This observation in Group 2 and Group 7, which covered the occipital areas, further supported the validity of the results as these are the areas where alpha was expected to be more evident.

Figure 6. Groupings of electroencephalogram channels.

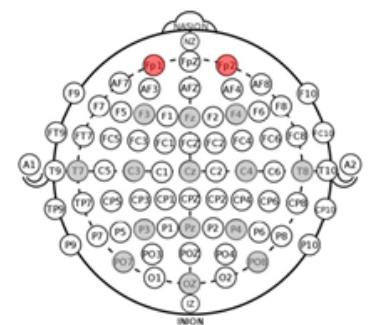

Group 1

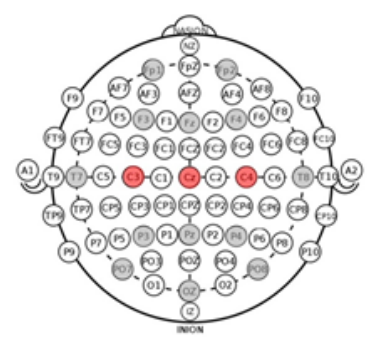

Group 4

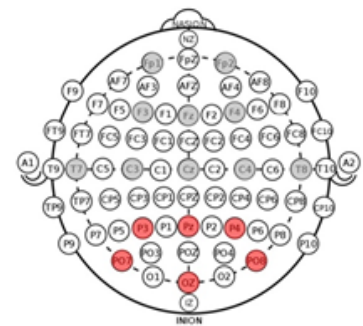

Group 7

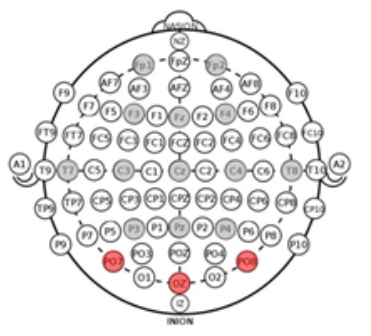

Group 2

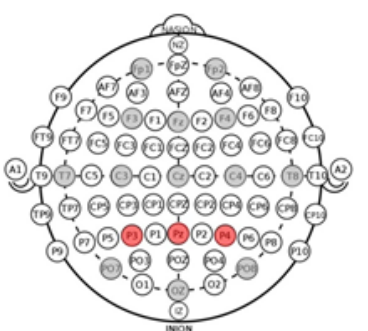

Group 5

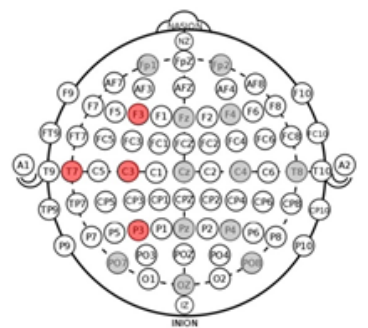

Group 8
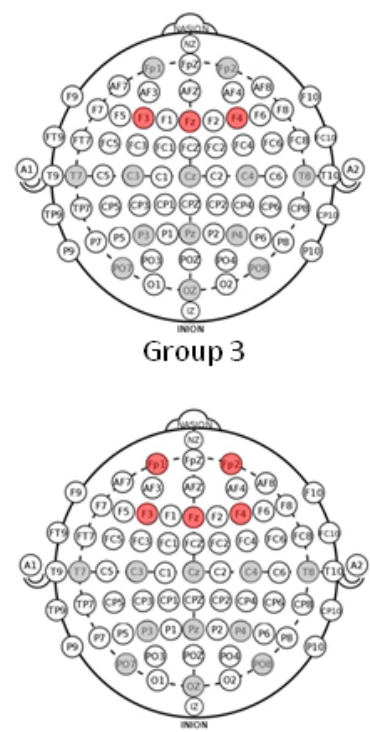

Group 6

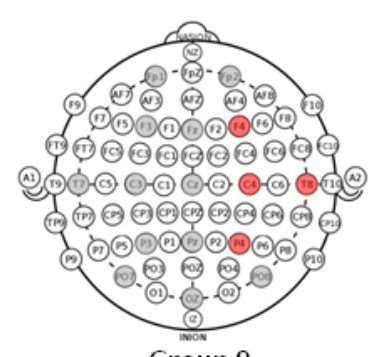

Group 9

\section{Discussion}

\section{Principal Results}

The goal of this study was to investigate whether brain training games with consumer-grade single-channel EEG sensor neurofeedback could be effective in enhancing the cognitive functions of elderly users. The results seemed to confirm its usefulness, especially in terms of memory improvements in both DMS and SWM. Compared to our prior works, SWM improvement was indeed also observed in NFT in children [30] and in our previous trial with older subjects [53]. The difference in terms of the setup between this trial and the previous trial [53] was that this trial used single-channel EEG instead of multichannel EEG (Emotiv Epoc). A single-channel EEG was more practical and easier to administer, but signal reading quality could suffer as a result. Another main difference is that this trial also employed the new feedback signal and adaptive threshold concepts. All of these factors could have contributed to the slightly different results obtained between the two studies.

For the attention domain, a significant change was found on MOT compared to significant changes found for RVP in previous studies [30,53]. This difference could also be due to the difference in population and setup. Notably, the MOT change was also found in the control group. As MOT was designed primarily for screening purposes, we could speculate that it might have a sensitivity that made it less suitable for attention assessment.

In general, the results of this study are in line with the current literature given a similar focus on two main areas of potential benefits: attention [61-63] and working memory [64,65]. Evidently, the published results to date are variable. For example, Gadea et al [66] reported no significant changes on 
theta downregulation but achieved significant changes in attention. By contrast, other studies [67-69] reported successful voluntary modulation of brain activity (change in the EEG signal) but no effects on the behavior (eg, symptom reduction). Both effects were shown in other studies [70-74]. A recent study concerning NFT using low-cost EEG found alpha enhancement but no effect on cognitive performance [75]. There are many factors that may have contributed to these mixed results. Experimental variables (factors) such as protocol, duration of the training, location of electrodes, or signal modality could have been set up differently, leading to these mixed results. Indeed, the large majority of neurofeedback studies appeared to have at least one major methodological limitation such as lack of randomization, nonblind designs, and use of waiting list control conditions. Controls without sham groups in a quasiexperimental design used in many trials means that evidence for the efficacy of the independent variable (ie, feedback training) could not be fully validated. Unfortunately, use of a sham control for the positive effects is ethically challenging, since the use of placebo (which also includes sham groups) instead of clinical treatments may lead to a deterioration of symptoms. Thus, the well-controlled EEG-based neurofeedback studies performed to date could only be carried out on treatment-resistant or healthy subjects. In nonblind designs, factors such as the relationship with the therapist are also relevant, especially when treating children.

Another issue regarding the effectiveness of NFT is sustainability validated through long-term studies. There have only been a few such studies, mostly targeted toward ADHD treatment. For example Lee et al [62] and Rijken et al [76] evaluated the long-term effect of NFT through follow-up. Compared to nonactive control treatments, NFT appeared to have more durable treatment effects for at least 6 months following treatment [77]. The reasons for the lack of such studies may be related to practical issues such as difficulty to reconcile schedules with the participants or withdrawal of subjects.

Despite these limitations, it is still evident from literature reviews that there is a clear scientific movement toward the use of neurofeedback as a tool to improve cognition and behavior besides rehabilitation. Moreover, it is interesting to note a shift in the direction of research in this field, characterized by a move from the classical clinical standard of evaluating the effectiveness of the method (standardized double-blind, randomized experiment) to the use of other assessment methods that seem more appropriate for neuropsychological treatments. The primary reason is related to contradictions between the positive outcomes of single case studies and the ineffectiveness of studies with large numbers of subjects [78]. This trend eventually may lead to the development of individualized treatment protocols, where effect of the treatment is assessed within the single case.

With respect to the details of the design, for simplicity, we used alpha high with a fixed frequency range, given recent studies indicating that it was possible to enhance cognitive capacities of healthy individuals by means of IUA NFT, where the IUA was located between the individual alpha peak (IAP; between 7.5 and $12.5 \mathrm{~Hz}$ ) and IAP+2 Hz [38]. Changes from upper alpha to IUA could have potential to improve the effectiveness further.
The training time of 30 minutes per session provided a user with considerable training time, while being short enough to not cause too much fatigue. During game development and testing, we found that an individual game duration of 2-3 minutes was appropriate. Game players usually spent the first 30 seconds up to 1 minute to ramp up their attention levels. These levels were then maintained or slightly improved throughout the game. After 2 minutes, the level of attention tended to drop, and several players felt fatigue and lost attention completely after 3 minutes. As a result, all 5 games were programmed to run for 2-3 minutes. Players were also asked to take a break after every game.

Each NFT set comprised a low-cost one-channel EEG headset and a PC with internet and Bluetooth connection. Once set up, the user could simply put on the headset and start a game. This could be a low-cost addition to community centers for the elderly around the country. These NFT games could be played leisurely to supplement existing activities such as group music and craft works.

Finally, as Staufenbiel et al [79] and Wang and Hsieh [80] pointed out, the literature remains sparse in relation to assessing the impact of neurofeedback in the elderly population. Our work, showing positive benefits on both EEG and cognitive functions, hopefully contributes as an additional step toward the confirmation of benefits for NFT in the elderly.

\section{Limitations}

This study has some limitations that warrant future research. First, the sample size could be considered to be small. According to Begemann et al [81], to detect a medium effect size of 0.5 , a minimal sample size of 64 per group would be needed. This criterion was not met by our study or most of the related works reported to date. Underpowered studies could carry the risk of both false-positive and false-negative findings, and are potentially more likely to be affected by publication bias, selective data analysis, and selective reporting of outcomes [82]. Moreover, as in the majority of similar research, our study design was quasiexperimental. This could also raise a question of how much the success observed in the modulation of the brain activity or behavior was due to actual training as opposed to nonspecific factors that could significantly contribute to the results. Although we have taken precautionary measures to limit these potential biases, a full randomized controlled trial with a higher number of samples would be one way to further solidify the findings. A long-term study would also be beneficial. To increase the chance for successful completion, the study could be designed with a shorter but more intensive intervention to encompass the follow-up phase with fewer possible withdrawals.

\section{Conclusions}

In this work, we have demonstrated the potential benefit of game-based brain exercise using neurofeedback. The system implemented was based on a practical single-channel EEG using new feedback and gaming techniques. Results from the 5-site pilot study have demonstrated improvement in the visual memory (DMS), attention (MOT), and visual recognition (SWM) domains. EEG analyses also showed improvement in 
upper alpha at a resting state (open-eyed) in the frontal area, which similarly indicated improvement in the cognitive domain.

\section{Acknowledgments}

The authors would like to acknowledge with gratitude the funding from the National Science and Technology Development Agency (NSTDA). The support we received from the staff of the NECTEC-CU Center of Technology for Cognitive Care in Elderly has also been very much appreciated. We are also thankful for local support by our partner sites, especially Sukunya Rojhprasert, PhD and Chanon Sirikul, MD from King Narai Hospital; Komwudh Koncha, MD from Queen Savang Vadhana Memorial Hospital; Mrs. Saowaluck Thanasirorat and Miss Phasita Hunarak from Thai Redcross Health Station 2; Miss Sasithon Thiengtham from Ban Bang Khae; and Mrs. Kulchalee Sukunwaranil from Thai Redcross Health Station 5.

\section{Conflicts of Interest}

None declared.

\section{References}

1. Multisectoral action for a life course approach to healthy ageing: draft global strategy and plan of action on ageing and health. 69th World Health Assembly, Geneva (A69/17). World Health Organization. 2016 Apr 22. URL: http://apps.who.int/ gb/ebwha/pdf files/WHA69/A69 17-en.pdf [accessed 2021-06-05]

2. Rao D, Luo X, Tang M, Shen Y, Huang R, Yu J, et al. Prevalence of mild cognitive impairment and its subtypes in community-dwelling residents aged 65 years or older in Guangzhou, China. Arch Gerontol Geriatr 2018;75:70-75. [doi: 10.1016/j.archger.2017.11.003] [Medline: 29197258]

3. Klimova B, Maresova P. Computer-based training programs for older people with mild cognitive impairment and/or dementia. Front Hum Neurosci 2017;11:262. [doi: 10.3389/fnhum.2017.00262] [Medline: 28559806]

4. Prince M, Bryce R, Albanese E, Wimo A, Ribeiro W, Ferri CP. The global prevalence of dementia: a systematic review and metaanalysis. Alzheimers Dement 2013 Jan;9(1):63-75. [doi: 10.1016/j.jalz.2012.11.007] [Medline: 23305823]

5. Bang J, Spina S, Miller BL. Frontotemporal dementia. Lancet 2015 Oct 24;386(10004):1672-1682 [FREE Full text] [doi: 10.1016/S0140-6736(15)00461-4] [Medline: 26595641]

6. Dementia Fact Sheet. World Health Organization. 2015. URL: http://www.who.int/mediacentre/factsheets/fs362/en/ [accessed 2021-06-05]

7. Wimo A, Winblad B, Jönsson L. The worldwide societal costs of dementia: Estimates for 2009. Alzheimers Dement 2010 Mar;6(2):98-103. [doi: 10.1016/j.jalz.2010.01.010] [Medline: 20298969]

8. Reisberg B, Ferris S, de Leon MJ, Crook T. Global Deterioration Scale (GDS). Psychopharmacol Bull 1988;24(4):661-663. [Medline: 3249768]

9. Fischer P, Jungwirth S, Zehetmayer S, Weissgram S, Hoenigschnabl S, Gelpi E, et al. Conversion from subtypes of mild cognitive impairment to Alzheimer dementia. Neurology 2007 Jan 23;68(4):288-291. [doi:

10.1212/01.wnl.0000252358.03285.9d] [Medline: 17242334]

10. Winblad B, Palmer K, Kivipelto M, Jelic V, Fratiglioni L, Wahlund L, et al. Mild cognitive impairment--beyond controversies, towards a consensus: report of the International Working Group on Mild Cognitive Impairment. J Intern Med 2004 Sep;256(3):240-246 [FREE Full text] [doi: 10.1111/j.1365-2796.2004.01380.x] [Medline: 15324367]

11. Petersen RC. Mild cognitive impairment as a diagnostic entity. J Intern Med 2004 Sep;256(3):183-194 [FREE Full text] [doi: 10.1111/j.1365-2796.2004.01388.x] [Medline: 15324362]

12. Alkhunizan M, Alkhenizan A, Basudan L. Prevalence of mild cognitive impairment and dementia in Saudi Arabia: a community-based study. Dement Geriatr Cogn Dis Extra 2018;8(1):98-103 [FREE Full text] [doi: 10.1159/000487231] [Medline: 29706986]

13. Mirza SS, Ikram MA, Bos D, Mihaescu R, Hofman A, Tiemeier H. Mild cognitive impairment and risk of depression and anxiety: A population-based study. Alzheimers Dement 2017 Feb;13(2):130-139. [doi: 10.1016/j.jalz.2016.06.2361] [Medline: 27520773]

14. Bae JB, Kim YJ, Han JW, Kim TH, Park JH, Lee SB, et al. Incidence of and risk factors for Alzheimer's disease and mild cognitive impairment in Korean elderly. Dement Geriatr Cogn Disord 2015;39(1-2):105-115. [doi: 10.1159/000366555] [Medline: 25401488]

15. Schneider LS, Dagerman KS, Insel P. Risk of death with atypical antipsychotic drug treatment for dementia: meta-analysis of randomized placebo-controlled trials. JAMA 2005 Oct 19;294(15):1934-1943. [doi: 10.1001/jama.294.15.1934] [Medline: $\underline{16234500]}$

16. Smith TGH. Antipsychotics in dementia--mortality risks and strategies to reduce prescribing. Evid Based Ment Health 2011 May;14(2):35-36. [doi: 10.1136/ebmh.14.2.35] [Medline: 21502142]

17. Vigen CL, Mack WJ, Keefe RS, Sano M, Sultzer DL, Stroup TS, et al. Cognitive effects of atypical antipsychotic medications in patients with Alzheimer's disease: outcomes from CATIE-AD. Am J Psychiatry 2011 Aug;168(8):831-839 [FREE Full text] [doi: 10.1176/appi.ajp.2011.08121844] [Medline: 21572163] 
18. Jalbert JJ, Daiello LA, Eaton CB, Miller SC, Lapane KL. Antipsychotic use and the risk of diabetes in nursing home residents with dementia. Am J Geriatr Pharmacother 2011 Jun;9(3):153-163. [doi: 10.1016/j.amjopharm.2011.04.006] [Medline: 21596626]

19. Coyle H, Traynor V, Solowij N. Computerized and virtual reality cognitive training for individuals at high risk of cognitive decline: systematic review of the literature. Am J Geriatr Psychiatry 2015 Apr;23(4):335-359. [doi: 10.1016/j.jagp.2014.04.009] [Medline: 24998488]

20. Hill NTM, Mowszowski L, Naismith SL, Chadwick VL, Valenzuela M, Lampit A. Computerized cognitive training in older adults with mild cognitive impairment or dementia: a systematic review and meta-analysis. Am J Psychiatry 2017 Apr 01;174(4):329-340. [doi: 10.1176/appi.ajp.2016.16030360] [Medline: 27838936]

21. Reijnders J, van Heugten C, van Boxtel M. Cognitive interventions in healthy older adults and people with mild cognitive impairment: a systematic review. Ageing Res Rev 2013 Jan;12(1):263-275. [doi: 10.1016/j.arr.2012.07.003] [Medline: 22841936]

22. Simon SS, Yokomizo JE, Bottino CM. Cognitive intervention in amnestic mild cognitive impairment: a systematic review. Neurosci Biobehav Rev 2012 Apr;36(4):1163-1178. [doi: 10.1016/j.neubiorev.2012.01.007] [Medline: 22322184]

23. Omejc N, Rojc B, Battaglini PP, Marusic U. Review of the therapeutic neurofeedback method using electroencephalography: EEG Neurofeedback. Bosn J Basic Med Sci 2019 Aug 20;19(3):213-220. [doi: 10.17305/bjbms.2018.3785] [Medline: 30465705]

24. Budzynski T. From EEG to neurofeedback. In: Invans JR, Arbarbanel A, editors. Introduction to Quantitative EEG Neurofeedback. 1st ed. San Diego: Academic Press Inc; 1999.

25. Neurofeedback Overview. International Society for Neuroregulation \& Research (ISNR). URL: https://www.isnr.org/ neurofeedback-introduction [accessed 2021-06-05]

26. Arns M, de Ridder S, Strehl U, Breteler M, Coenen A. Efficacy of neurofeedback treatment in ADHD: the effects on inattention, impulsivity and hyperactivity: a meta-analysis. Clin EEG Neurosci 2009 Jul;40(3):180-189. [doi: 10.1177/155005940904000311] [Medline: 19715181]

27. Schoenberg PLA, David AS. Biofeedback for psychiatric disorders: a systematic review. Appl Psychophysiol Biofeedback 2014 Jun;39(2):109-135. [doi: 10.1007/s10484-014-9246-9] [Medline: 24806535]

28. Sonuga-Barke EJS, Brandeis D, Cortese S, Daley D, Ferrin M, Holtmann M, European ADHD Guidelines Group. Nonpharmacological interventions for ADHD: systematic review and meta-analyses of randomized controlled trials of dietary and psychological treatments. Am J Psychiatry 2013 Mar;170(3):275-289. [doi: 10.1176/appi.ajp.2012.12070991] [Medline: 23360949]

29. Sudnawa KK, Chirdkiatgumchai V, Ruangdaraganon N, Khongkhatithum C, Udomsubpayakul U, Jirayucharoensak S, et al. Effectiveness of neurofeedback versus medication for attention-deficit/hyperactivity disorder. Pediatr Int 2018 Sep;60(9):828-834. [doi: 10.1111/ped.13641] [Medline: 29931709]

30. Israsena P, Hemrungrojn S, Sukwattanasinit N, Maes M. Development and evaluation of an interactive electro-encephalogram-based neurofeedback system for training attention and attention defects in children. J Med Imaging Hlth Inform 2015 Sep 01;5(5):1045-1052. [doi: 10.1166/jmihi.2015.1492]

31. Hammond DC. Neurofeedback with anxiety and affective disorders. Child Adolesc Psychiatr Clin N Am 2005 Jan;14(1):105-123. [doi: 10.1016/j.chc.2004.07.008] [Medline: 15564054]

32. Wan L, Huang H, Schwab N, Tanner J, Rajan A, Lam NB, et al. From eyes-closed to eyes-open: Role of cholinergic projections in EC-to-EO alpha reactivity revealed by combining EEG and MRI. Hum Brain Mapp 2019 Feb 01;40(2):566-577 [FREE Full text] [doi: 10.1002/hbm.24395] [Medline: $\underline{\text { 30251753] }}$

33. Reis J, Portugal A, Pereira M, Dias N. Alpha and theta intensive neurofeedback protocol for age related cognitive deficits. 2015 Presented at: 7th International IEEE/EMBS Conference on Neural Engineering (NER); April 22-24, 2015; Montpellier, France p. 715. [doi: 10.1109/NER.2015.7146723]

34. Navarro Gil M, Escolano Marco C, Montero-Marín J, Minguez Zafra J, Shonin E, García Campayo J. Efficacy of neurofeedback on the increase of mindfulness-related capacities in healthy individuals: a controlled trial. Mindfulness 2017 Jul 14;9(1):303-311. [doi: 10.1007/s12671-017-0775-1]

35. Hsueh J, Chen T, Chen J, Shaw F. Neurofeedback training of EEG alpha rhythm enhances episodic and working memory. Hum Brain Mapp 2016 Jul;37(7):2662-2675 [FREE Full text] [doi: 10.1002/hbm.23201] [Medline: 27038114]

36. Klimesch W. EEG-alpha rhythms and memory processes. Int J Psychophysiol 1997 Jun;26(1-3):319-340. [doi: 10.1016/s0167-8760(97)00773-3] [Medline: 9203012]

37. Angelakis E, Lubar JF, Stathopoulou S, Kounios J. Peak alpha frequency: an electroencephalographic measure of cognitive preparedness. Clin Neurophysiol 2004 Apr;115(4):887-897. [doi: 10.1016/j.clinph.2003.11.034] [Medline: 15003770]

38. Klimesch W. EEG alpha and theta oscillations reflect cognitive and memory performance: a review and analysis. Brain Res Brain Res Rev 1999 Apr;29(2-3):169-195. [doi: 10.1016/s0165-0173(98)00056-3] [Medline: 10209231]

39. Nan W, Rodrigues JP, Ma J, Qu X, Wan F, Mak P, et al. Individual alpha neurofeedback training effect on short term memory. Int J Psychophysiol 2012 Oct;86(1):83-87. [doi: 10.1016/j.ijpsycho.2012.07.182] [Medline: 22864258]

40. Escolano C, Aguilar M, Minguez J. EEG-based upper alpha neurofeedback training improves working memory performance. Annu Int Conf IEEE Eng Med Biol Soc 2011;2011:2327-2330. [doi: 10.1109/IEMBS.2011.6090651] [Medline: 22254807] 
41. Posthuma D, Neale MC, Boomsma DI, de Geus EJ. Are smarter brains running faster? Heritability of alpha peak frequency, IQ, and their interrelation. Behav Genet 2001 Nov;31(6):567-579. [doi: 10.1023/a:1013345411774] [Medline: 11838534]

42. Stankova EP, Myshkin IY. Association between individual EEG characteristics and the level of intelligence. Moscow Univ Biol Sci Bull 2016 Dec 28;71(4):256-261. [doi: 10.3103/s0096392516040118]

43. Lias S, Murat Z, Sulaiman N, Taib M. IQ Index using alpha-beta correlation of EEG power spectrum density (PSD). : IEEE; 2010 Presented at: IEEE Symposium on Industrial Electronics and Applications (ISIEA); October 3-5, 2010; Penang, Malaysia p. 612-616. [doi: 10.1109/isiea.2010.5679391]

44. Begemann MJH, Florisse EJR, van Lutterveld R, Kooyman M, Sommer IE. Efficacy of EEG neurofeedback in psychiatry: A comprehensive overview and meta-analysis. Transl Brain Rhythmicity 2016;1(1):19-29. [doi: 10.15761/tbr.1000105]

45. Rogala J, Jurewicz K, Paluch K, Kublik E, Cetnarski R, Wróbel A. The do's and don'ts of neurofeedback training: a review of the controlled studies using healthy adults. Front Hum Neurosci 2016;10:301. [doi: 10.3389/fnhum.2016.00301] [Medline: 27378892]

46. American Electroencephalographic Society. American Electroencephalographic Society guidelines for standard electrode position nomenclature. J Clin Neurophysiol 1991 Apr;8(2):200-202. [Medline: 2050819]

47. Ratti E, Waninger S, Berka C, Ruffini G, Verma A. Comparison of medical and consumer wireless EEG systems for use in clinical trials. Front Hum Neurosci 2017;11:398. [doi: 10.3389/fnhum.2017.00398] [Medline: 28824402]

48. Sawangjai P, Hompoonsup S, Leelaarporn P, Kongwudhikunakorn S, Wilaiprasitporn T. Consumer grade EEG measuring sensors as research tools: a review. IEEE Sensors J 2020 Apr 15;20(8):3996-4024. [doi: 10.1109/jsen.2019.2962874]

49. Khng KH, Mane R. Beyond BCI-Validating a wireless, consumer-grade EEG headset against a medical-grade system for evaluating EEG effects of a test anxiety intervention in school. Adv Eng Informat 2020 Aug;45:101106. [doi: 10.1016/j.aei.2020.101106]

50. Taherian S, Selitskiy D, Pau J, Claire Davies T. Are we there yet? Evaluating commercial grade brain-computer interface for control of computer applications by individuals with cerebral palsy. Disabil Rehabil Assist Technol 2017 Feb;12(2):165-174. [doi: 10.3109/17483107.2015.1111943] [Medline: 26699697]

51. Maskeliunas R, Damasevicius R, Martisius I, Vasiljevas M. Consumer-grade EEG devices: are they usable for control tasks? PeerJ 2016;4:e1746. [doi: 10.7717/peerj.1746] [Medline: 27014511]

52. Grummett TS, Leibbrandt RE, Lewis TW, DeLosAngeles D, Powers DMW, Willoughby JO, et al. Measurement of neural signals from inexpensive, wireless and dry EEG systems. Physiol Meas 2015 Jul;36(7):1469-1484. [doi:

10.1088/0967-3334/36/7/1469] [Medline: 26020164]

53. Jirayucharoensak S, Israsena P, Pan-Ngum S, Hemrungrojn S, Maes M. A game-based neurofeedback training system to enhance cognitive performance in healthy elderly subjects and in patients with amnestic mild cognitive impairment. Clin Interv Aging 2019;14:347-360. [doi: 10.2147/CIA.S189047] [Medline: 30863028]

54. Unity Technologies. URL: https://unity.com/ [accessed 2021-06-05]

55. Marzbani H, Marateb HR, Mansourian M. Neurofeedback: a comprehensive review on system design, methodology and clinical applications. Basic Clin Neurosci 2016 Apr;7(2):143-158 [FRE Full text] [doi: 10.15412/J.BCN.03070208] [Medline: 27303609]

56. Liang S, Lin C, Wu R, Chen Y, Huang T, Jung T. Monitoring driver's alertness based on the driving performance estimation and the EEG power spectrum analysis. 2005 Presented at: IEEE Engineering in Medicine and Biology 27th Annual Conference; January 17-18, 2006; Shanghai, China p. 5738-5741. [doi: 10.1109/iembs.2005.1615791]

57. Hamadicharef B, Zhang H, Guan C, Wang C, Phua K, Tee K, et al. Learning eeg-based spectral-spatial patterns for attention level measurement. 2009 Presented at: IEEE International Symposium on Circuits and Systems; May 24-27, 2009; Taipei, Taiwan p. 1465-1468. [doi: 10.1109/iscas.2009.5118043]

58. Li Y, Li X, Ratcliffe M, Liu L, Qi Y, Liu Q. A real-time EEG-based BCI system for attention recognition in ubiquitous environment. 2011 Presented at: 2011 International Workshop on Ubiquitous Affective Awareness and Intelligent Interaction; September 2011; Beijing, China p. 33-40. [doi: 10.1145/2030092.2030099]

59. Chun MM, Turk-Browne NB. Interactions between attention and memory. Curr Opin Neurobiol 2007 Apr;17(2):177-184. [doi: 10.1016/j.conb.2007.03.005] [Medline: 17379501 ]

60. Egner T, Gruzelier JH. EEG biofeedback of low beta band components: frequency-specific effects on variables of attention and event-related brain potentials. Clin Neurophysiol 2004 Jan;115(1):131-139. [doi: 10.1016/s1388-2457(03)00353-5] [Medline: $\underline{14706480]}$

61. Zandi Mehran Y, Firoozabadi M, Rostami R. Improvement of neurofeedback therapy for improved attention through facilitation of brain activity using local sinusoidal extremely low frequency magnetic field exposure. Clin EEG Neurosci 2015 Apr;46(2):100-112. [doi: 10.1177/1550059414524403] [Medline: 24939868]

62. Lee T, Quek SY, Goh SJA, Phillips R, Guan C, Cheung YB, et al. A pilot randomized controlled trial using EEG-based brain-computer interface training for a Chinese-speaking group of healthy elderly. Clin Interv Aging 2015;10:217-227. [doi: 10.2147/CIA.S73955] [Medline: 25624754]

63. Yeo SN, Lee TS, Sng WT, Heo MQ, Bautista D, Cheung YB, et al. Effectiveness of a personalized brain-computer interface system for cognitive training in healthy elderly: a randomized controlled trial. J Alzheimers Dis 2018;66(1):127-138. [doi: 10.3233/JAD-180450] [Medline: $\underline{\text { 30248056] }}$ 
64. Reis J, Portugal AM, Fernandes L, Afonso N, Pereira M, Sousa N, et al. An alpha and theta intensive and short neurofeedback protocol for healthy aging working-memory training. Front Aging Neurosci 2016;8:157. [doi: 10.3389/fnagi.2016.00157] [Medline: 27458369]

65. Campos da Paz VK, Garcia A, Campos da Paz Neto A, Tomaz C. SMR neurofeedback training facilitates working memory performance in healthy older adults: a behavioral and EEG study. Front Behav Neurosci 2018;12:321. [doi: 10.3389/fnbeh.2018.00321] [Medline: 30618671]

66. Gadea M, Aliño M, Garijo E, Espert R, Salvador A. Testing the benefits of neurofeedback on selective attention measured through dichotic listening. Appl Psychophysiol Biofeedback 2016 Jun;41(2):157-164. [doi: 10.1007/s10484-015-9323-8] [Medline: 26683198]

67. Naas A, Rodrigues J, Knirsch J, Sonderegger A. Neurofeedback training with a low-priced EEG device leads to faster alpha enhancement but shows no effect on cognitive performance: A single-blind, sham-feedback study. PLoS One 2019;14(9):e0211668 [FREE Full text] [doi: 10.1371/journal.pone.0211668] [Medline: 31483789]

68. Gonçalves Ó, Carvalho S, Mendes AJ, Leite J, Boggio PS. Neuromodulating attention and mind-wandering processes with a single session real time EEG. Appl Psychophysiol Biofeedback 2018 Jun;43(2):143-151. [doi: 10.1007/s10484-018-9394-4] [Medline: 29797155]

69. Sherwood MS, Parker JG, Diller EE, Ganapathy S, Bennett KB, Esquivel CR, et al. Self-directed down-regulation of auditory cortex activity mediated by real-time fMRI neurofeedback augments attentional processes, resting cerebral perfusion, and auditory activation. Neuroimage 2019 Jul 15;195:475-489 [FREE Full text] [doi: 10.1016/j.neuroimage.2019.03.078] [Medline: 30954710$]$

70. Zoefel B, Huster RJ, Herrmann CS. Neurofeedback training of the upper alpha frequency band in EEG improves cognitive performance. Neuroimage 2011 Jan 15;54(2):1427-1431. [doi: 10.1016/j.neuroimage.2010.08.078] [Medline: 20850552]

71. Hoedlmoser K, Pecherstorfer T, Gruber G, Anderer P, Doppelmayr M, Klimesch W, et al. Instrumental conditioning of human sensorimotor rhythm $(12-15 \mathrm{~Hz})$ and its impact on sleep as well as declarative learning. Sleep 2008 Oct;31(10):1401-1408 [FREE Full text] [Medline: 18853937]

72. Gevensleben H, Holl B, Albrecht B, Vogel C, Schlamp D, Kratz O, et al. Is neurofeedback an efficacious treatment for ADHD? A randomised controlled clinical trial. J Child Psychol Psychiatry 2009 Jul;50(7):780-789. [doi:

10.1111/j.1469-7610.2008.02033.x] [Medline: 19207632]

73. Steiner NJ, Frenette EC, Rene KM, Brennan RT, Perrin EC. In-school neurofeedback training for ADHD: sustained improvements from a randomized control trial. Pediatrics 2014 Mar;133(3):483-492. [doi: 10.1542/peds.2013-2059] [Medline: 24534402]

74. Strehl U, Leins U, Goth G, Klinger C, Hinterberger T, Birbaumer N. Self-regulation of slow cortical potentials: a new treatment for children with attention-deficit/hyperactivity disorder. Pediatrics 2006 Nov;118(5):e1530-e1540. [doi: 10.1542/peds.2005-2478] [Medline: 17060480]

75. Naas A, Rodrigues J, Knirsch J, Sonderegger A. Neurofeedback training with a low-priced EEG device leads to faster alpha enhancement but shows no effect on cognitive performance: A single-blind, sham-feedback study. PLoS One 2019;14(9):e0211668 [FREE Full text] [doi: 10.1371/journal.pone.0211668] [Medline: $\underline{31483789}$ ]

76. Rijken NH, Soer R, de Maar E, Prins H, Teeuw WB, Peuscher J, et al. Increasing performance of professional soccer players and elite track and field athletes with peak performance training and biofeedback: a pilot study. Appl Psychophysiol Biofeedback 2016 Dec;41(4):421-430 [FREE Full text] [doi: 10.1007/s10484-016-9344-y] [Medline: 27761664]

77. Van Doren J, Arns M, Heinrich H, Vollebregt MA, Strehl U, K Loo S. Sustained effects of neurofeedback in ADHD: a systematic review and meta-analysis. Eur Child Adolesc Psychiatry 2019 Mar;28(3):293-305 [FREE Full text] [doi: 10.1007/s00787-018-1121-4] [Medline: 29445867]

78. Rossiter T. The effectiveness of neurofeedback and stimulant drugs in treating AD/HD: Part I. Review of methodological issues. Appl Psychophysiol Biofeedback 2004 Jun;29(2):95-112. [doi: 10.1023/b:apbi.0000026636.13180.b6]

79. Staufenbiel SM, Brouwer A, Keizer AW, van Wouwe NC. Effect of beta and gamma neurofeedback on memory and intelligence in the elderly. Biol Psychol 2014 Jan;95:74-85. [doi: 10.1016/j.biopsycho.2013.05.020] [Medline: 23751914]

80. Wang J, Hsieh S. Neurofeedback training improves attention and working memory performance. Clin Neurophysiol 2013 Dec;124(12):2406-2420. [doi: 10.1016/j.clinph.2013.05.020] [Medline: 23827814]

81. Begemann MJH, Florisse EJR, van Lutterveld R, Kooyman M, Sommer IE. Efficacy of EEG neurofeedback in psychiatry: A comprehensive overview and meta-analysis. Transl Brain Rhythmicity 2016;1(1):19-29. [doi: 10.15761/tbr.1000105]

82. Button KS, Ioannidis JPA, Mokrysz C, Nosek BA, Flint J, Robinson ESJ, et al. Power failure: why small sample size undermines the reliability of neuroscience. Nat Rev Neurosci 2013 May;14(5):365-376. [doi: 10.1038/nrn3475] [Medline: $\underline{23571845]}$

\section{Abbreviations}

ADHD: attention deficit hyperactivity disorder

API: application programming interface

CANTAB: Cambridge Neuropsychological Test Automated Battery 
DMS: delayed matching to sample

EEG: electroencephalogram

IAP: individual alpha peak

IUA: individual upper alpha

MCI: mild cognitive impairment

MoCA: The Montreal Cognitive Assessment

MOT: motor screening task

NFT: neurofeedback training

PSD: power spectral density

RVP: rapid visual information processing

SWM: spatial working memory

TMSE: Thai Mental State Examination

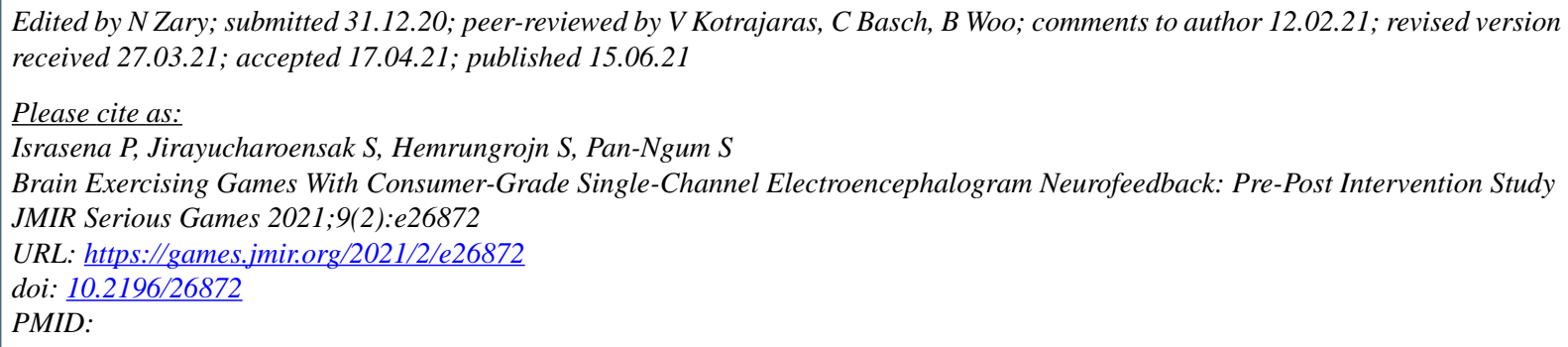

CPasin Israsena, Suwicha Jirayucharoensak, Solaphat Hemrungrojn, Setha Pan-Ngum. Originally published in JMIR Serious Games (https://games.jmir.org), 15.06.2021. This is an open-access article distributed under the terms of the Creative Commons Attribution License (https://creativecommons.org/licenses/by/4.0/), which permits unrestricted use, distribution, and reproduction in any medium, provided the original work, first published in JMIR Serious Games, is properly cited. The complete bibliographic information, a link to the original publication on https://games.jmir.org, as well as this copyright and license information must be included. 